\title{
Modelling of blood coagulation in cerebral aneurysms
}

\author{
Y. Aida $^{1}$ \& K Shimano ${ }^{2}$ \\ ${ }^{1}$ Graduate School, Tokyo City University, Japan \\ ${ }^{2}$ Department of Mechanical Systems Engineering, \\ Tokyo City University, Japan
}

\begin{abstract}
A fatal subarachnoid haemorrhage occurs when an intracranial aneurysm ruptures and there are no guidelines for rupture risk assessment with perfect reliability. Thrombus formation in stagnant flow condition has been said to play an important role in rupture. However, there are, at present, no mathematical models that allow us to simulate the whole process of thrombus formation in aneurysms. In this study, the authors proposed a mathematical model of intraaneurysmal coagulation so that the amount of fibrin generated in the aneurysm can be calculated. It is known that coagulation is initiated by erythroelastase-IX (EE-IX) in stagnant flow condition. This process is different from contact and tissue factor pathways. In the present coagulation model, the reaction rate constant of factor IX activation by EE-IX was determined. A group of reactions in which anticoagulants are involved are treated as a whole so as to reduce computational cost, and a quantity for the combined reaction rate constant is also determined. It is demonstrated that the present coagulation model can reproduce the rate of fibrin generation in a reference experiment at a reasonable computational cost.

Keywords: intracranial aneurysm, rupture, stagnant blood flow, coagulation, erythroelastase-IX.
\end{abstract}

\section{Introduction}

Intracranial aneurysms cause fatal subarachnoid haemorrhages when they rupture. Unruptured aneurysms can be found by means of magnetic resonance imaging (MRI) or computed tomography (CT) before the patients have any 
symptoms, and there are neurosurgical treatments to prevent future rupture of the detected aneurysms, e.g. clipping and coiling. However, such a treatment is not applied to every aneurysm because not all aneurysms rupture and because the treatments are so invasive [1] that the patients' lives could be jeopardised. It is, therefore, necessary to make a critical decision about whether to apply any treatment whenever an unruptured aneurysm is found. Although it seems reasonable to treat only aneurysms with high probabilities of future rupture, it is difficult with the present understanding to predict precisely which aneurysms are likely to rupture.

Two promising morphological parameters, aspect ratio (AR) and size ratio (SR), have been proposed for rupture prediction. It is considered that an aneurysm with a higher AR or SR is more likely to rupture than one with a lower AR or SR. AR is a ratio of aneurysmal dome depth to neck width while SR is defined as a ratio of dome length to parent vessel diameter. Ujiie et al. [2] reported a significant correlation of AR with rupture status and proposed a criterion $\mathrm{AR}>1.6$ for assessing rupture risk. Other researchers [3, 4] concurred with this correlation. A comparably high correlation between SR and rupture status was also reported [5-7] and it was suggested that SR $>2$ could be a criterion for rupture risk assessment.

A haemodynamical significance of AR and SR is slowness of intraaneurysmal flow. Shimano et al. [8] theoretically clarified that AR and SR are factors directly affecting velocity scale of the intra-aneurysmal flow. In stagnant flow condition, thrombus formation is likely. Ujiie et al. [9] suggested that thrombi play an important role in degeneration of the artery wall because of the effect of the enzyme involved in ensuing fibrinolysis. In an experiment of Takahashi et al. [10], thrombus formation was observed in a model aneurysm with an AR of 2.3. Shimano et al. [11, 12], who applied their platelet aggregation model to two model aneurysms, found that stagnant flow in aneurysmal domes prompts active platelet aggregation. For further understanding of the issue, it is necessary to develop a computational model which allows us to simulate the whole process of intra-aneurysmal thrombus formation in consideration of not only platelet aggregation but also coagulation.

Coagulation is a process in which insoluble fibrin is generated at the end of a cascade of enzymatic reactions. In stagnant flow, the coagulation cascade is initiated by erythroelastase-IX (EE-IX) [13], an enzyme found on membranes of red blood cells (RBCs). This process is not identical to the tissue factor pathway (extrinsic pathway) or the contact pathway (intrinsic pathway). Referring to an experiment by Iwata et al. [13], the authors propose a mathematical model of coagulation initiated by EE-IX so that the amount of fibrin generated in the aneurysm can be evaluated.

The present coagulation model is expressed in the form of a system of ordinary differential equations with derivatives of time. However, when the model is applied to blood flow in a real aneurysm, spatial derivatives representing the convection and diffusion should be added to the model equations. In this context, it is necessary to assure not only high accuracy but also low computational cost. 
There are so many anticoagulants that it would require a high computational cost to consider all anticoagulants. Furthermore, it is even impossible to do so with some anticoagulants of which effects have not been fully elucidated. Thus, reactions related to anticoagulants are treated jointly with other reactions. This means that a group of closely connected reactions are considered as a whole by deriving a combined reaction rate constant. Determination of such combined reaction rate constants is a task of primary importance in this study.

Another important task is determination of the rate of factor-IX activation by EE-IX because its quantity is unknown.

Details of the present coagulation model are discussed in this paper. Unless otherwise stated, each coagulation factor is represented by capital letter $\mathrm{F}$ followed by the corresponding Roman numeral, e.g. F-IX. Roman numerals combined with "a" mean that the coagulation factors are activated.

\section{Thrombus formation in aneurysms}

\subsection{Coagulation initiated by erythroelastase-IX}

Neither contact (intrinsic) nor tissue factor (extrinsic) pathway is responsible for coagulation in cerebral aneurysms because the walls of unruptured aneurysms are usually intact. According to Takahashi et al. [10], the time-scale of thrombus formation in an endothelialised model aneurysm was approximately 1020 minutes while the time-scale is tens of seconds in normal haemostasis with the tissue factor pathway. Coagulation in cerebral aneurysms is, therefore, considered to occur in the same manner as venous thrombus formation.

It has been clarified that activation of F-IX by erythroelastase-IX (EE-IX) initiates coagulation of the venous thrombus formation [13]. The coagulation cascade initiated by EE-IX is depicted schematically in Fig. 1 where each reaction is given a unique ID number. As shown in the legend, there are two types of arrows in Fig. 1: arrows pointing at substances represent the transformation of substrates to products and those pointing at the middle of other arrows indicate enzymatic actions. Substances in the rectangles are anticoagulants. Reactions $1-3,15$ and 16 constitute the main structure of the cascade while the other reactions represent feedback, acceleration and inhibition. In the present model, reactions are divided into groups, each of which is treated as a whole (see chapter 3 for details). Some groups are shown in Fig. 1 by dashed lines but not all groups appear in the figure to avoid overlaying too great a number of dashed lines.

F-IX is activated by EE-IX through reaction 1 only when a level of shear rate is extremely low (less than 10 1/s). It was reported by Kaibara [14] that the rate of the F-IX activation is higher under a lower shear rate. The resultant substance F-IXaEE is basically the same as normal F-IXa but 10 times slower in activation of F-X [13]. Reactions after activation of F-X are identical to those in the tissue factor pathway. Coagulation induced by EE-IX is slower than in normal haemostasis mainly because reaction 1 is extremely slow. However, the rate of 
fibrin generation is determined by not only the initial reaction but also a balance between the accelerating and decelerating effects explained below.

A feedback mechanism increases the rate of activation of F-X via F-XIa and F-IXa (reactions 5, 7, 8 and 9). On the other hand, there are two acceleration mechanisms via F-V and F-VIII, respectively. More about the acceleration mechanisms will be explained in section 2.2.

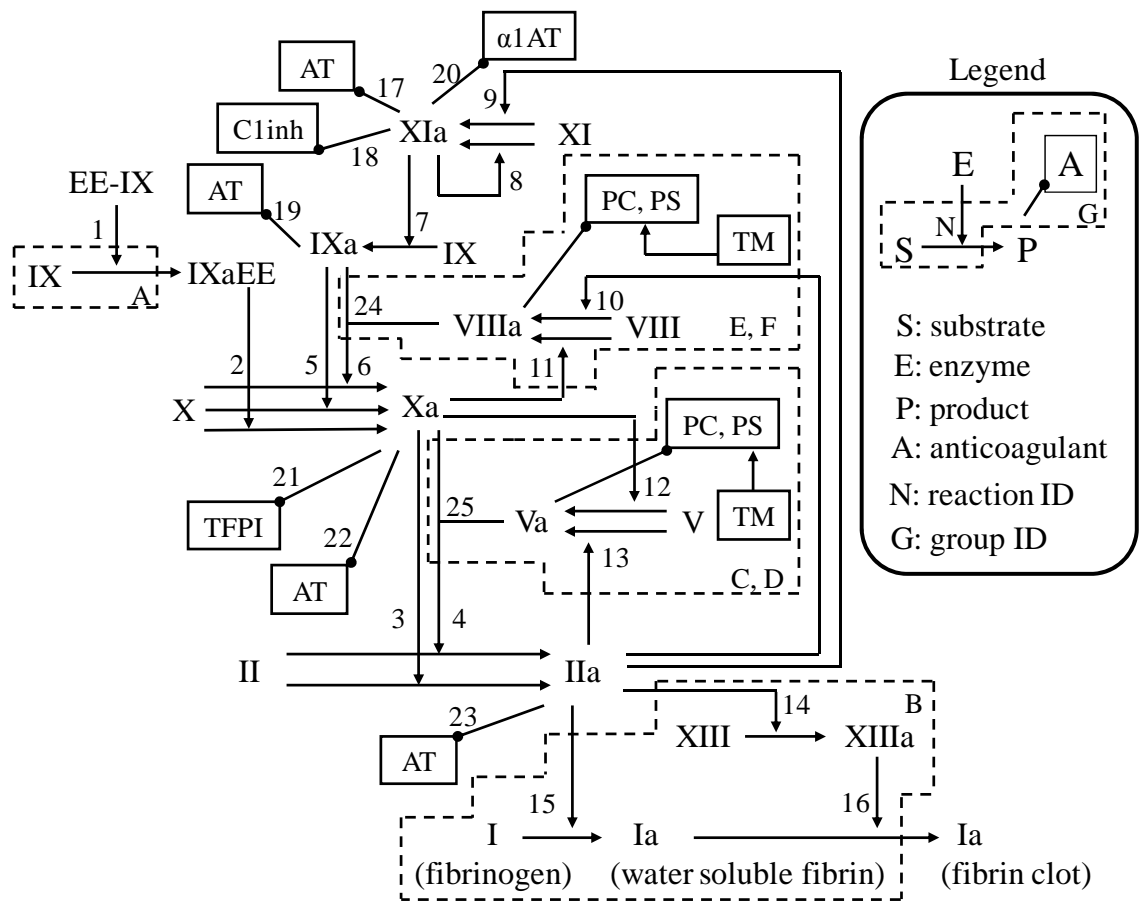

Figure 1: The coagulation cascade initiated by erythroelastase-IX. Capital letter " $F$ " representing coagulation factors is omitted for simplicity.

There are various anticoagulants, i.e. antithrombin-III (AT) [15, 16], C1inhibitor (C1inh) [17], tissue factor pathway inhibitor (TFPI) [18], protein-C (PC) [19], protein-S (PS) [20], thrombomodulin (TM) [21] and $\alpha 1$-antitrypsin $(\alpha$ 1AT) [22]. Although TM forms a complex with F-IIa before activating PC, formation of the complex is not depicted in Fig. 1 for transparency.

\subsection{Acceleration mechanisms}

Two acceleration mechanisms are illustrated in Fig. 2. Both mechanisms are closely connected to platelets. When F-IIa (thrombin) is generated through reaction 3, as shown in Fig. 1, F-IIa activates F-VIII and F-V through reactions 10 and 13, respectively. Furthermore, F-IIa induces platelet aggregation which allows two complexes, tenase and prothrombinase, to form on the surfaces of the 
activated platelets. Tenase [23], a complex of F-IXa and F-VIIIa, accelerates activation of F-X (reaction 6) and, consequently, contributes to quicker generation of thrombin (reaction 3). Prothrombinase [24], a complex of F-Xa and F-Va, has a similar effect through reaction 4: F-IIa is generated more quickly from F-II (prothrombin) in the presence of prothrombinase. F-IIa generated through these acceleration mechanisms contributes to activation of still more platelets.

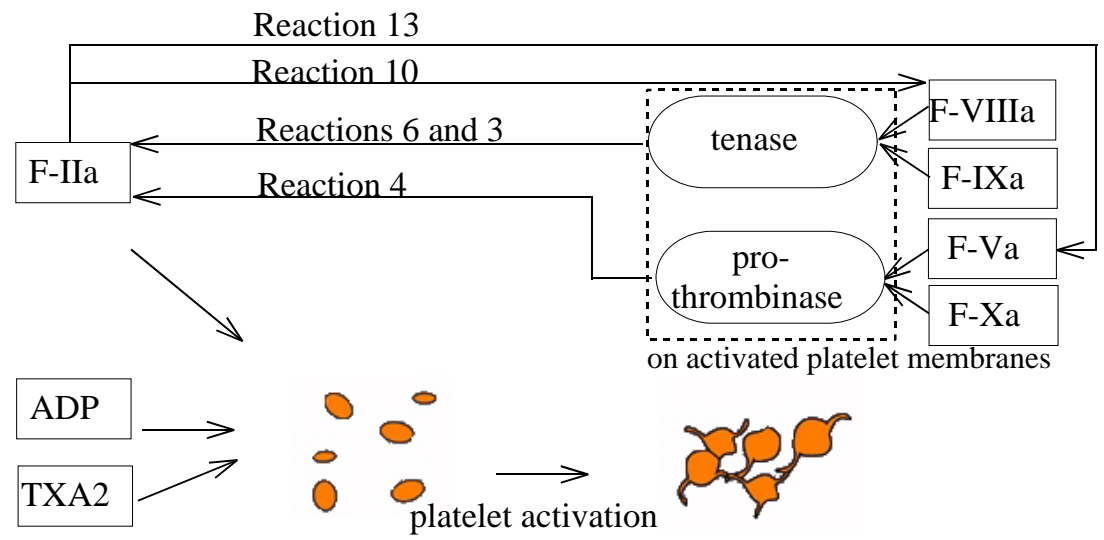

Figure 2: $\quad$ Acceleration mechanisms via tenase and prothrombinase.

Not only F-IIa but also ADP (adenosine diphosphate) and TXA2 (thromboxane A2) can activate platelets. When ADP or TXA2 is released into plasma for some reason, the acceleration mechanisms explained above have a stronger effect. In fact, this can happen in intra-aneurysmal flow.

Figure 3 shows velocity vectors in a model aneurysm investigated by Shimano et al. [12]. It can be seen in the figure that the incoming flow approaching the entrance to the aneurysm hits the edge of the aneurysmal neck.

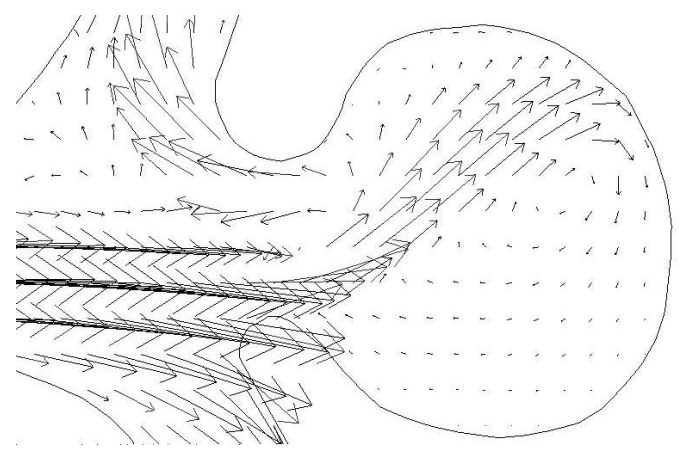

Figure 3: Velocity vectors on the central cross section of a model aneurysm at the systole calculated by Shimano et al. [12]. 
RBCs and platelets receive shearing force due to a large velocity gradient near the impingement point. A level of the shear stress reaches approximately 10 times as high as that exerted on normal artery walls. Such mechanical stimuli can cause escape of cell contents including ADP and TXA2 into the surrounding plasma. It can be concluded that intra-aneurysmal coagulation tends to be quicker than pure venous coagulation due to ADP and TXA2 originating from RBCs and platelets.

The acceleration mechanisms related to F-IIa are considered in the present coagulation model. However, the effect of ADP or TXA2 is not included because a reference experiment [13] used here was carried out with whole blood free from strong shearing force.

\section{Modelling of coagulation initiated by EE-IX}

\subsection{Approach}

When an enzyme acts on a substrate, the product is generated with concentration of the enzyme unchanged. The reaction rate is expressed by Michaelis-Menten kinetics. The rate of change in concentration of the product is written in the following form:

$$
\frac{d[P]}{d t}=k_{c}[E][S]
$$

where $[P],[E]$ and $[S]$ are concentrations of the product, enzyme and substrate, respectively, and $k_{c}$ is the reaction rate constant. The term on the left hand side of eqn (1) represents the rate of increase in $[P]$, which is identical to the rate of decrease in $[S]$.

Anticoagulants form complexes by trapping some coagulation factors which are, consequently, prevented from being activated. The process of the complex formation is reversible: the complexes decompose at the same time. These twoway reactions can also be described by multiplying the corresponding reaction rate constant to the product of the reactant concentrations.

By applying the strategy explained above, a system of ordinary differential equations is obtained for concentrations of all the substances shown in Fig. 1. However, the equations are not solved one by one. For the sake of reduction in computational cost, the reactions are divided into groups and those in each group are treated as a whole with a combined reaction rate considered.

Strictly, it is impossible to define concentration for EE-IX because it is on RBC membranes. In this study, equivalent concentration is calculated for EE-IX from haematocrit.

\subsection{Reaction rate constants}

Reaction rate constants are listed in Table 1 for all the reaction groups. Combined reaction rates for groups G-L are evaluated by Lineweaver-Burk plot from rate constants of individual reactions, which are cited from Hockin et al. [25] and Jones et al. [26]. 
Table 1: Grouping of reactions and corresponding reaction rate constants. Reaction IDs are the same as in Fig. 1. LB stands for LineweaverBurk.

\begin{tabular}{|c|c|r|l|}
\hline Group & Reaction IDs & $\begin{array}{c}\text { Reaction rate } \\
\text { constant }\end{array}$ & $\begin{array}{c}\text { Method of } \\
\text { evaluation }\end{array}$ \\
\hline A & 1 & $5.0 \times 10^{2} \mathrm{M}^{-1} \mathrm{~s}^{-1}$ & Ref. to experiment \\
\hline B & $14,15,16$ & $2.5 \times 10^{3} \mathrm{M}^{-1} \mathrm{~s}^{-1}$ & Ref. to experiment \\
\hline C & 12,25 & $0 \mathrm{M}^{-1} \mathrm{~s}^{-1}$ & Ref. to experiment \\
\hline D & 13,25 & $9.0 \times 10^{4} \mathrm{M}^{-1} \mathrm{~s}^{-1}$ & Ref. to experiment \\
\hline E & 10,24 & $7.1 \times 10^{2} \mathrm{M}^{-1} \mathrm{~s}^{-1}$ & Ref. to experiment \\
\hline F & 11,24 & $0 \mathrm{M}^{-1} \mathrm{~s}^{-1}$ & Ref. to experiment \\
\hline G & $2,21,22$ & $8.5 \times 10^{2} \mathrm{M}^{-1} \mathrm{~s}^{-1}$ & LB plot \\
\hline H & $5,21,22$ & $8.5 \times 10^{3} \mathrm{M}^{-1} \mathrm{~s}^{-1}$ & LB plot \\
\hline $\mathrm{I}$ & $6,21,22$ & $9.3 \times 10^{7} \mathrm{M}^{-1} \mathrm{~s}^{-1}$ & LB plot \\
\hline $\mathrm{J}$ & 3,23 & $6.3 \times 10^{5} \mathrm{M}^{-1} \mathrm{~s}^{-1}$ & LB plot \\
\hline K & 4,23 & $1.4 \times 10^{5} \mathrm{M}^{-1} \mathrm{~s}^{-1}$ & LB plot \\
\hline L & $7,8,9,17,18,19,20$ & $1.7 \times 10^{4} \mathrm{M}^{-1} \mathrm{~s}^{-1}$ & LB plot \\
\hline
\end{tabular}

Group A represents the initiation of the coagulation cascade. No quantitative information about the reaction rate constant of F-IX activation by EE-IX has been reported. The authors, therefore, referred to an experimental result of Iwata et al. [13] to determine it. In their experiment, change in a logarithmic damping factor (LDF), which represents change in blood viscosity, was measured by a damped oscillation rheometer. It was assumed that the initial and final values of LDF obtained in the experiment corresponded to 0 and $100 \%$ concentrations of fibrin, respectively, and that a linear mapping between LDF and fibrin concentration was applicable. Iwata's experimental result is shown in Fig. 4 with a dashed line. Here, a concentration of $100 \%$ indicates the state that all fibrinogen (F-I) contained in plasma $(7 \mu \mathrm{M})$ has become fibrin (F-Ia).

There are some anticoagulants of which quantitative information has not been fully elucidated, i.e. PC, PS and TM. Exclusion of these anticoagulants from the model would result in inaccurate computation with too quick generation of fibrin because they play an important role in retarding the acceleration mechanisms. For compensation of this, the combined rate constants of the groups $(\mathrm{C}-\mathrm{F})$, in which these anticoagulants are involved, are determined by trial-and-error with reference to Iwata's experiment.

Groups $\mathrm{C}$ and $\mathrm{D}$ represent acceleration through prothrombinase: $\mathrm{F}-\mathrm{V}$ is activated by F-Xa in group $\mathrm{C}$ and by F-IIa in D. It was found that change in the constant for group $\mathrm{C}$ does not cause any significant change in the computational result. This is presumably because F-IIa has a much stronger effect on activation of F-V than F-Xa. It is, therefore, assumed that the contribution of group $\mathrm{C}$ is negligible. For exactly the same reason, group $\mathrm{F}$ is also neglected.

Reaction 16 producing insoluble fibrin (F-Ia) is a cross-linking reaction for which eqn (1) cannot account. Thus, this reaction is put into group B together 
with reactions 14 and 15, and a combined reaction rate for group B is evaluated in the same manner as groups $\mathrm{A}$ and $\mathrm{C}-\mathrm{F}$.

The shape of the fibrin concentration curve (the dashed line in Fig. 4) is characterised by (a) the temporal point of the initial rise, (b) the high rate of increase and (c) the temporal point of the convergence. Groups A and B are mainly responsible for (a) and (c) respectively, while (c) is sensitive to choices of the rate constants for groups D and E. In other words, there are only weak interactions among the groups of which rate constants are determined from the experimental result. The trial-and-error process on each group was, therefore, able to be conducted almost independently.

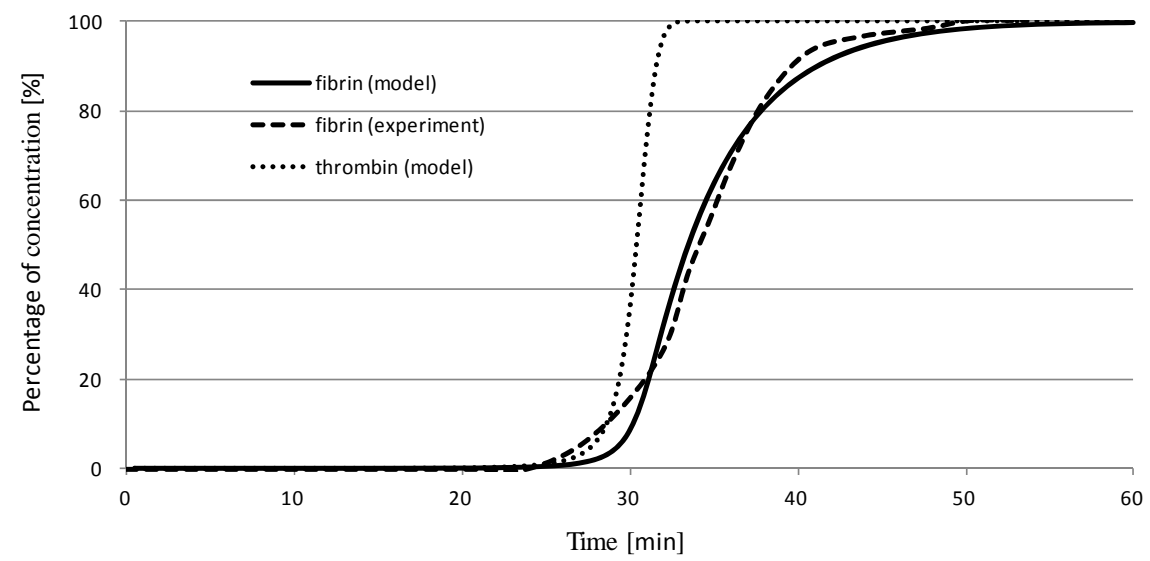

Figure 4: $\quad$ Histories of fibrin (F-Ia) and thrombin (F-IIa) concentrations. Each concentration is expressed as a percentage to the initial concentration of the substrate.

\section{Results and discussion}

The model equations were numerically solved by an explicit time-marching scheme. Time-step $\Delta t$ was as small as $0.001 \mathrm{~s}$ and no $\Delta t$-dependency of the computational result was observed when compared with $\Delta t=0.0005$. Computation was carried out up to 60 minutes (3.6 million time-steps).

EE-IX exists on RBC membranes, not in plasma. Thus, a quantity equivalent to EE-IX concentration is considered. The initial equivalent concentration was calculated with an assumption of $40 \%$ haematocrit. Concentrations of the other substances in normal plasma were used as the initial conditions.

Temporal change in fibrin (F-Ia) and thrombin (F-IIa) concentrations calculated with the present model are shown in Fig. 4 alongside the experimental result by Iwata et al. [13]. Each concentration is expressed as a percentage to the initial concentration of the corresponding substrate: $100 \%$ means a state that the substrate has completely transformed to the product. 
The initial rise in fibrin concentration is observed at approx. 25 minutes in both computational and experimental results. This is a natural result because the reaction rate constant of EE-IX was determined so that the timing of the initial rise could be precisely reproduced.

Although fibrin is generated even before 25 minutes, the order of magnitude of fibrin concentration is so small that any increase cannot be seen in Fig. 4 during that period. Hence, $t=25 \mathrm{~min}$. is regarded as a temporal point when the degree of fibrin generation reaches a perceivable level. Reactions 1 and 2 are responsible for this extremely slow progress in the early stage. However, once a significant amount of thrombin (F-IIa) has been generated, the feedback and acceleration mechanisms causes a "chain reaction" and results in rapid generation of fibrin (F-Ia). It is clearly seen in Fig. 4 that a sharp rise in thrombin (F-IIa) concentration is immediately followed by a rapid increase in fibrin concentration.

A fairly good agreement on the fibrin concentration between the experiment and simulation is observed throughout the coagulation process although minor gaps in gradient appear immediately after the initial rise and before the convergence. These differences in gradient can be attributed to the approximation made in the grouping.

As discussed in the last paragraph of the previous chapter, the fibrin concentration curve is characterised by (a) the temporal point of the initial rise, (b) the high rate of increase and (c) the temporal point of the convergence. All these features can be explained in connection with properties of blood and the related reactions: activation of F-IX by EE-IX is greatly responsible for (a), a balance between the acceleration mechanisms and inhibition by anticoagulants accounts for (b), and the degree of the cross-linking reaction from soluble to insoluble fibrin is correlated to (c). Therefore, determination of reaction rate constants by trial-and-error in this study is not a mere fitting to the experimental result but a biochemically meaningful evaluation. For example, the present model could adapt to blood with a higher haematocrit by choosing a larger value for group A's reaction rate constant. For blood with weaker anticoagulants, larger reaction rate constants would be given to groups $\mathrm{D}$ and $\mathrm{E}$.

The grouping strategy enables reduction in computational cost: it is expected that the computational time would almost double for a coagulation model with a one-by-one treatment of all the substrates and anticoagulants. It can be concluded that a good balance of computational cost and accuracy is realised with the present coagulation model.

The computational result produced by the present coagulation model reflects the effect of platelets activated by F-IIa (thrombin) but not that of platelets activated by ADP or TXA2 because the model is based on an in-vitro experiment with whole blood free from strong shearing force. When the model is applied to real cerebral aneurysms, contributions of ADP and TXA2 to the acceleration mechanisms should be taken into consideration. 


\section{Conclusion}

Considering past studies which suggest a correlation of thrombus formation with rupture of intracranial aneurysms, the authors developed a mathematical model which describes coagulation in cerebral aneurysms. Reaction rate constants were determined by trial-and-error so that the model could reproduce the same rate of fibrin generation as in a reference experiment. Reduction in the computational cost was also achieved by combining several chemical reactions. Although further studies are necessary for modelling of the effect of ADP and TXA2 originating from RBCs and platelets, the present coagulation model is expected to provide useful insights into thrombus formation in cerebral aneurysms when applied to intra-aneurysmal blood flow.

\section{References}

[1] The International Study of Unruptured Intracranial Aneurysms Investigators, Unruptured Intracranial Aneurysms - Risk of Rupture and Risks of Surgical Intervention, New England Journal of Medicine, 339(24), pp. 1725-1731, 1998.

[2] Ujiie, H., Tamano, Y., Sasaki, K. and Hori, T., Is the aspect ratio a reliable index for predicting the rupture of a saccular aneurysm? Neurosurgery, 48(3), pp. 495-503, 2001.

[3] Weir, B., Amidei, C., Kongable, G., Findlay, J.M., Kassell, N.F., Kelly, J., Dai, L. and Karrison, T.G., The aspect ratio (dome/neck) of ruptured and unruptured aneurysms, Journal of Neurosurgery, 99(3), pp. 447-451, 2003.

[4] Nader-Sepahi, A., Casimiro, M., Sen, J. and Kitchen, N.D., Is Aspect Ratio A reliable Predictor of Intracranial Aneurysm Rupture? Neurosurgery, 54(6), pp. 1343-1348, 2004.

[5] Dhar, S., Tremmel, M., Mocco, J., Kim, M., Yamamoto, J., Siddiqui, A.H., Hopkins, L.N. and Meng, H., Morphology Parameters for Intracranial Aneurysm Rupture Risk Assessment, Neurosurgery, 63(2), pp. 185-197, 2008.

[6] Tremmel, M., Dhar, S., Levy, E.I., Mocco, J. and Meng, H., Influence of Intracranial Aneurysm-to-Parent Vessel Size Ratio on Hemodynamics and Implication for Rupture: Results from a Virtual Experimental Study, Neurosurgery, 64(4), pp. 622-631, 2009.

[7] Rahman, M., Smietana, J., Hauck, E., Hoh, B., Hopkins, N., Siddiqui, A., Levy, E.I., Meng, H. and Mocco, J., Size Ratio Correlates With Intracranial Aneurysm Rupture Status (A Prospective Study), Stroke, 41, pp. 916-920. 2010.

[8] Shimano, K., Aida, Y. and Nakagawa, Y., Slowness of flow and resultant thrombus formation in cerebral aneurysms, Journal of Biorheology, 24(2), pp. 47-55, 2010.

[9] Ujiie, H., Tachibana, H., Hiramatsu, O., Hazel, A.L., Matsumoto, T., Ogasawara, Y., Nakajima, H., Hori, T., Takakura, K. and Kajiya, F., Effects of size and shape (aspect ratio) on the hemodynamics of saccular 
aneurysms: a possible index for surgical treatment of intracranial aneurysms, Neurosurgery, 45(1), pp. 110-130, 1999.

[10] Takahashi, N., Ujiie, H., Yotoriyama, T., Suzuki, Y., Hori, T. and Kaibara, M., Flow visualization study of the endothelialized glass aneurysm model implanting canine carotid artery (in Japanese with English abs.), Journal of Japanese Society of Biorheology, 18(4), pp. 143-148, 2004.

[11] Shimano, K., Ujiie, H. and Enomoto, Modeling of Platelet aggregation in Cerebrovascular Bifurcation Aneurysms, IFMBE Proc. vol. 25, World Congress on Medical Physics\& Biomedical Engineering, Springer, pp. 495-498, 2009.

[12] Shimano, K., Kudo, T., Yoshimoto, S., Ujiie, H., and Enomoto, Y., A Numerical study of Blood Flow Patterns in Cerebral Aneurysms and the Causal Relationship with Platelet Aggregation, International Journal of Design \& Nature and Ecodynamics, 5(2), pp. 122-141, 2010.

[13] Iwata, H., Kaibara, M., Dohmae, N., Takio, K., Himeno, R. and Kawakami S., Purification, identification, and characterization of elastase on erythrocyte membrane as factor IX-activating enzyme, Biochemical and Biophysical Research Communications, 316(1), pp. 65-70, 2004.

[14] Kaibara, M., Rheological study on coagulation of blood with special reference to the triggering mechanism of venous thrombus formation, Journal of Biorheology, 23(1), pp. 2-10, 2009.

[15] Jordan, R.E., Oosta, G.M., Gardner, W.T. and Rosenberg, RD., The kinetics of hemostatic enzyme-antithrombin interactions in the presence of low molecular weight heparin, The Journal of Biological Chemistry, 255, pp. 10081-10090, 1980.

[16] Schoen, P. and Lindhout, T., The in situ inhibition of prothrombinaseformed human alpha-thrombin and meizothrombin(des F1) by antithrombin III and heparin, The Journal of Biological Chemistry, 262 , pp. 1126811274, 1987.

[17] Bouma B.N. and Griffin J.H., Initiation Mechanism: the contact activation system in plasma, Blood Coagulation, eds. Zwaal R.F.A. and Hemker H.C., Elsevier: Amsterdam, pp. 103-128, 1986.

[18] Baugh, R.J., Broze, G.J., Jr. and Krishnaswamy, S., Regulation of extrinsic pathway factor Xa formation by tissue factor pathway inhibitor, The Journal of Biological Chemistry, 273, pp. 4378-4386, 1998.

[19] Anand M, Rajagopal K, Rajagopal KR , A model for the formation, growth and lysis of clots in quiescent plasma: a comparison between the effects of antithrombin III deficiency and protein C deficiency, Journal of Theoretical Biology, 253(4), pp. 725-738, 2008.

[20] Heeb, M.J., Mesters, R.M., Tans, G., Rosing, J. and Griffin, J.H., Binding of protein $\mathrm{S}$ to factor $\mathrm{Va}$ associated with inhibition of prothrombinase that is independent of activated protein C, The Journal of Biological Chemistry, 268, pp. 2872-2877, 1993.

[21] Griffin, J.H., Control of coagulation reactions (Chapter 107). Topics in Williams Hematology, Seventh Edition, eds. M.A. Lichtman, E. Beutler, 
T.J. Kipps, U. Seligsohn, K. Kaushansky and J.T. Prchal, The McGraw-Hill Companies: New York, pp. 1695-1714, 2006.

[22] Gettins, P.G.W., Serpin Structure, Mechanism, and Function, Chemical Reviews, 102, pp. 4751-4804, 2002.

[23] Varadi K. and Hemker H.C., Kinetics of the Formation of the Factor X Activating Enzyme of the Blood Coagulation System, Thrombosis Research, 8(3), pp. 303-317, 1976.

[24] Rosing J., Tans G., Govers-Riemslag J.W.P., Zwaal R.F.A. and Hemker H.C., The Role of Phospholipids and Factor Va in the Prothrombinase Complex, The Journal of Biological Chemistry, 255, pp. 274-283, 1980.

[25] Hockin, M.F., Jones, K.C., Everse, S.J. and Mann, K.G., A Model for the Stoichiometric Regulation of Blood Coagulation, The Journal of Biological Chemistry, 277, pp. 18322-18333, 2002.

[26] Jones, K.C. and Mann K.G., A model for the tissue factor pathway to thrombin. II. A mathematical simulation, The Journal of Biological Chemistry, 269, pp. 23367-23373, 1994. 